\title{
Heroismus und Defätismus: alte und neue Feindbilder in den Chansons der Poilus
}

Héroïsme et défaitisme : anciennes et nouvelles perceptions de l'ennemi dans les chansons des Poilus

Heroism and Defeatism: Old and New Perceptions of the Enemy in the Poilus

Songs

Janina Arndts

\section{OpenEdition \\ Journals}

Édition électronique

URL : http://journals.openedition.org/ceg/1979

DOI : $10.4000 /$ ceg. 1979

ISSN : 2605-8359

Éditeur

Presses Universitaires de Provence

\section{Édition imprimée}

Date de publication : 15 juin 2014

Pagination : 93-104

ISSN : 0751-4239

Référence électronique

Janina Arndts, "Heroismus und Defätismus: alte und neue Feindbilder in den Chansons der Poilus », Cahiers d'Études Germaniques [Online], 66 | 2014, Online erschienen am: 17 Dezember 2017, abgerufen am 19 November 2020. URL : http://journals.openedition.org/ceg/1979 ; DOI : https://doi.org/ $10.4000 /$ ceg. 1979 


\title{
Heroismus und Defätismus Alte und neue Feindbilder in den Chansons der Poilus
}

\author{
Janina ARNDTS \\ Aix-Marseille/Tübingen
}

Die äußerst konfliktreiche Geschichte der deutsch-französischen Beziehungen hat über viele Generationen hinweg tiefe Spuren im kollektiven Gedächtnis beider Nationen hinterlassen. Das von häufigen kriegerischen Auseinandersetzungen beeinträchtigte Verhältnis zwischen Deutschen und Franzosen spiegelt sich auch im Liedgut beider Völker wider. Es existieren französische Chansons, die über viele Jahrzehnte den Hass auf die Deutschen propagieren, sowie deutsche Lieder, die einen virulenten Franzosenhass verbreiten. Ebenso wie die Kriege zwischen beiden Nationen formen von Ressentiments und Revanchegedanken geprägte Lieder, Hymnen und Chansons das kollektive Gedächtnis beider Völker. Frankophobe Lieder werden in Deutschland, germanophobe Chansons in Frankreich über die Elternhäuser, den Schulunterricht, die Gesangsvereine und den Militärdienst an die jeweils nächste Generation vermittelt. Ein solches Liedgut perpetuiert und verstärkt die gegenseitige Konfliktbereitschaft und wird mit jedem Konflikt immer umfangreicher. Bevor der Frage nachgegangen wird, ob es Abweichungen von diesem musikalischen Mainstream gibt, sollen hier zunächst die Traditionslinien eines Liedguts aufgezeigt werden, das Feindbilder kultiviert.

\section{Erste Feindbilder}

Auf der deutschen Seite entstehen die ersten frankophoben Lieder während der Zeit der Befreiungskriege, vor allem in den Jahren 1812 und 1813. Zu diesen Liedern gehört zum Beispiel Ernst Moritz Arndts Der Gott, der Eisen wachsen ließ, ein Text, der in seiner martialischen Phantasie davon träumt, mit „Franzosenblut" das „Eisen (zu) röten“. 
Weitere frankophobe Lieder entstehen während der Rheinkrise von 1840/41. Hier sind das Rheinlied von Nikolaus Becker und Die Wacht am Rhein von Max Schneckenburger zu nennen, während das von Hoffmann von Fallersleben gedichtete Lied der Deutschen entgegen einer geläufigen Einschätzung und trotz der Zeile Deutschland, Deutschland über alles kein frankophobes Lied ist.

Nikolaus Becker widmete sein im September 1840 veröffentlichtes Rheinlied dem Dichter Alphonse de Lamartine, der im Januar 1840 in einer Rede vor der französischen Kammer den Anspruch Frankreichs auf das linke Rheinufer erneuert hatte. Das Lied wird so zu einer zugeeigneten Replik. Es beginnt mit folgenden Zeilen: „Sie sollen ihn nicht haben / den freien deutschen Rhein, ob sie wie gierige Raben / sich heiser danach schrein." Die anlässlich der Rheinkrise geschriebenen Verse wurden von Robert Schumann vertont und bald überall gesungen. Reichskanzler Otto von Bismarck sagte 1893 über das Rheinlied, dass es wegen der Schnelligkeit, mit der es von der Bevölkerung aufgegriffen wurde, wirkte, ,als ob wir ein paar Armeecorps mehr am Rhein stehen hätten, als wir hatten" " Alphonse de Lamartine antwortete am 1. Juni 1841 mit seiner Marseillaise de la Paix, der einen Tag später Alfred de Mussets Le Rhin allemand folgte. Musset erinnert in seinem später vertonten Gedicht an die gegen Ludwig XIV. und Napoleon I. erlittenen militärischen Niederlagen der Deutschen: „Nous l'avons eu, votre Rhin allemand. Que faisaient vos vertus germaines, Quand notre César toutpuissant / De son ombre couvrait vos plaines?“".

Als ausgesprochen frankophobes Lied kann Die Wacht am Rhein bezeichnet werden, in der es unter anderem heißt: „Solang ein Tropfen Blut noch glüht / noch eine Faust den Degen zieht, und noch ein Arm die Büchse spannt / betritt kein Welscher deinen Strand. Lieb Vaterland, magst ruhig sein / Fest steht und treu die Wacht, die Wacht am Rhein!“. Das Lied Die Wacht am Rhein hatte im Kaiserreich von 1871 neben der Hymne Heil dir im Siegerkranz beim Volk den - wenngleich nie staatlich anerkannten - Status einer Nationalhymne der Deutschen; das Lied wurde häufig bei offiziellen Anlässen gesungen.

Auch wenn die Rheinkrise militärisch folgenlos blieb, beweist sie doch, wie groß auf beiden Seiten des Rheins die Bereitschaft war, einen deutschfranzösischen Krieg zu riskieren. Die patriotischen und meist frankophoben Lieder der Befreiungskriege und der Rheinkrise gehörten zum musikalischen Marschgepäck der Preussen, Badener, Bayern und Württemberger, die 1870 in den Deutsch-Französischen Krieg zogen. Diese Lieder gehörten ebenfalls zum musikalischen Repertoire der deutschen Soldaten, die im August 1914 in den Ersten Weltkrieg marschierten. Hinzu kamen nun Lieder, die den Sieg von 1870, insbesondere die Schlacht von Sedan, den Kaiser und Bismarck

\footnotetext{
Zitiert in Aachener Zeitung vom 2. Oktober 2009: http://www.aachenerzeitung.de/lokales/geilenkirchen/nikolaus-becker-gluehender-patriot-oder-dummer-kerl1.328920 (Zugriff 27.08.2013).
} 
glorifizierten. In den Tornistern der Deutschen befanden sich auch Kriegsliederbücher. Eines von ihnen trug den bezeichnenden Titel Jeder Sto $\beta$ ein Franzos ${ }^{2}$ und kündigte damit die Bajonettkämpfe an, die auf den Schlachtfeldern des Ersten Weltkriegs bevorstanden.

Im kollektiven Gedächtnis Frankreichs kann der Deutsch-Französische Krieg von 1870/71 und der Erste Weltkrieg eine Einheit bilden, insofern den Söhnen und Enkeln der in Sedan und Metz besiegten Franzosen 1918 die lang ersehnte Revanche gegen das Deutsche Reich gelingt.

Das musikalische Marschgepäck der Poilus bestand zu Beginn des Ersten Weltkrieges aus der Marseillaise, dem Chant du départ und den nach der Niederlage im Deutsch-Französischen Krieg verfassten chansons revanchardes.

Die von Rouget de Lisle in der Nacht auf den 26. April 1792 während der Kriegserklärung an Österreich im elsässischen Straßburg verfasste Marseillaise hatte zunächst den Titel Chant de guerre pour l'armée du RhinKriegslied für die Rheinarmee. Mit den Worten Aux armes, citoyens, formez vos bataillons! rief die Marseillaise zum Kampf gegen die Feinde Frankreichs auf, damit - wie es blutrünstig heißt - deren unreines Blut der Äcker Furchen tränke. Das Kriegslied wurde 1795 erstmals und 1871 erneut zur Nationalhymne Frankreichs erklärt.

Das Revolutions- und Kriegslied Le Chant du Départ war von 1804 bis 1815 die Nationalhymne Frankreichs und Schlachtgesang der napoleonischen Heere. Das mit den Zeilen La victoire en chantant nous ouvre la barrière beginnende Lied blieb bis in unsere Zeit populär.

\section{La chanson revancharde}

Als getreues Abbild einer nationalistisch gesinnten Gesellschaft entsteht ab 1871 ein neues musikalisches Genre, das sich bis zum Beginn des Ersten Weltkrieges beim französischen Publikum großer Beliebtheit erfreut: la chanson revancharde. Wegen des enormen Erfolges solcher Chansons spezialisieren sich Autoren und Komponisten wie Lucien Delormel, Gaston Villemer und Paul Déroulède, die selbst am verlorenen Krieg teilgenommen haben, auf die Produktion revanchistischer Lieder.

Den Reigen der chansons revanchardes eröffnet Gaston Villemers 1871 veröffentlichtes Chanson Vous n'aurez pas l'Alsace et la Lorraine ! Es thematisiert eines der Leitmotive der französischen Politik der Nachkriegszeit, nämlich die Rückeroberung der beiden verlorenen Provinzen. Dieses Ziel wird einer der Gründe für die Teilnahme Frankreichs am Ersten Weltkrieg sein. Der Refrain des Chansons ist eine patriotische Kampfansage der Elsässer und Lothringer an die Deutschen: « Vous n'aurez pas l'Alsace et

\footnotetext{
2 Irmgard HeIDLER, Der Verleger Eugen Diederichs und seine Welt (1896-1930), Wiesbaden, Harrassowitz Verlag, 1998, S. 393.
} 
la Lorraine, / Et, malgré vous, nous resterons français, / Vous avez pu germaniser la plaine, / Mais notre cour vous ne l'aurez jamais ! ».

Heroisierende Chansons erleichtern die gesellschaftliche Bewältigung der militärischen Niederlage von 1871, indem sie die Tapferkeit der besiegten Soldaten besingen, diese Tugend aber den an Zahl und Material überlegenen Siegern absprechen. Im Repertoire der chanson revancharde folgen vor allem die Chansons Les Cuirassiers de Reichshoffen und Le Clairon diesem aus politischer und psychologischer Sicht verständlichen Schema: Das negative Feindbild beider Chansons soll das nationale Selbstbild aufwerten.

Die überwiegend deutschsprachigen Elsass-Lothringer teilen häufig die in Frankreich virulente haine $d u$ Boche nicht. Viele bleiben Frankreich emotional verbunden, können aber Deutschland, das ihnen kulturell nahe steht, nicht hassen ${ }^{3}$. Die Autoren der chansons revanchardes setzen sich über dieses Dilemma der Elsass-Lothringer hinweg: Sie erfinden Geschichten, in denen sich Männer, Frauen und sogar Kinder hasserfüllt gegen die deutsche Fremdherrschaft wehren. Einige Beispiele seien hier genannt.

Als in Frankreich die Befürchtung wächst, dass die ledigen Frauen der verlorenen Provinzen auch preußische Soldaten heiraten könnten, nimmt sich Villemer 1881 dieser Thematik in seinem Chanson La fiancée alsacienne an. Eine junge Elsässerin liebt ihren in der französischen Armee dienenden Verlobten so sehr, dass sie sich eher das Leben nimmt, als sich von einem preußischen Offizier zur Hochzeit zwingen zu lassen.

Besonders aufschlussreich ist das Chanson Le fils de l'Allemand, dessen Text Villemer und Delormel 1882 verfassen: Ein preußischer Offizier klopft an die Tür einer Lothringerin, die gerade ihr eigenes Kind stillt. Er bringt ihr seinen Sohn, dessen Mutter einen Tag zuvor bei der Geburt gestorben ist, und fleht sie an, dem Säugling von ihrer Milch zu geben. Die Frau lehnt diese Bitte ab: Im Krieg hätten preußische Soldaten ihren erstgeborenen Sohn in seiner Wiege getötet. Ihre Muttermilch sei französisch: "Mes garçons chanteront plus tard la Marseillaise, / Je ne vends pas mon lait au fils d'un Allemand. » Das Thema der Revanche ist auch in diesem Chanson präsent: Zum einen verweigert die Lothringerin den Ammendienst, zum anderen verkündet sie, dass ihre Söhne eines Tages die Marseillaise singen werden. Die Darstellung der Preußen als barbarische Soldateska, die selbst Kinder in der Wiege tötet, soll die Franzosen in ihrer haine du Boche bestärken und der Patriotismus der lothringischen Mutter sie zur Revanche verpflichten.

Diese doppelte Funktion erfüllt auch das 1885 geschriebene Chanson Le violon brisé. Ein Greis erzählt, dass die Kinder gerade zum Spiel seiner Violine tanzten, als unerwartet die Feinde erschienen. Als er ohne Angst auf die Deutschen zugegangen sei, hätten die Soldaten ihn zu Boden gestoßen und dann gefragt, wer er denn sei, um ihnen die Stirn zu bieten. Nach seiner Antwort „Je suis, dis-je, en me redressant / L'ennemi des peuples esclaves !“

\footnotetext{
3 Alfred WAHL, Jean-Claude RichEZ, L'Alsace entre France et Allemagne, Paris, Hachette, 1994, S. 250.
} 
hätten sie ihn geohrfeigt und verlangt, die Lieder ihres Königs zu spielen. Als er stattdessen die Marseillaise angestimmt habe, hätten die Deutschen seine Violine zerbrochen: Die zerbrochene Violine soll verdeutlichen, dass die Deutschen nicht länger zu den Kultur-völkern zählen. Sie gehören vielmehr zu den peuples esclaves, die ihren Monarchen untertan sind und die Ideale der Französischen Revolution - Liberté, Égalité, Fraternité - bekämpfen. Nicht anders als zur Zeit der Revolutionskriege, ist die Marseillaise, die der Greis spielt, eine gegen die äußeren Feinde Frankreichs und seiner Ideale gerichtete Hymne.

Andere Chansons zeigen explizit, dass von der neuen Armee der Dritten Republik die Revanche für die 1870/71 erlittene Demütigung erwartet wird : In seinem Chanson En revenant de la revue beschreibt Delormel die Militärparade, die anlässlich des französischen Nationalfeiertages am 14. Juli 1886 vor den Toren von Paris in Longchamp stattfindet. Dieses Chanson bleibt bis 1914 eines der beliebtesten Lieder zum Ruhme der französischen Armee.

1889 fordert Delormel die Soldaten der Dritten Republik auf, sich an den militärischen Siegen der Napoleonischen Armee ein Beispiel zu nehmen. In seinem Chanson Le Père la Victoire erzählt ein hundertjähriger Kriegsveteran einer Gruppe junger Rekruten sein Soldatenleben. Die Rekruten sollten ihm nacheifern: "Marchez à la gloire ! / Mes chers enfants, / Revenez triomphants. » Mit diesem Appell ruft der Kriegsveteran die Rekruten zur Revanche auf.

An die aus französischer Sicht glorreiche Zeit der Napoleonischen Kriege erinnert auch das Chanson Le Rêve passe aus dem Jahre 1906: Einem jungen Soldaten erscheint im Traum zunächst die Kavallerie Napoleons, dann eine Hydra, die auf jedem ihrer neun Köpfe eine preußische Pickelhaube trägt. Im Kampf gegen die preußische Hydra sollen die Soldaten der Dritten Republik ebenso tapfer sein wie die napoleonischen Helden. Die Metapher, die den deutschen Feind als schlangenartiges Ungeheuer karikiert, ist der griechischen Mythologie entlehnt. Im Repertoire der chansons revanchardes stellt diese Karikatur eine weitere Variante des propagierten Feindbildes dar. Die französischen Soldaten sollen - wie einst der Sagenheld Herakles - die Hydra enthaupten.

\section{Chansons der Grande Guerre}

Die Chansons, die das Heldentum der Poilus der Grande Guerre besingen, entstehen fern der Front. Ihre Pariser Autoren kennen das Kriegsgeschehen oft nur aus den offiziellen Frontberichten. Ihre Lieder werden an der Front kaum gesungen. Roland Dorgelès schildert in seinem 1919 erschienenen Antikriegsroman Les Croix de bois mehrere Szenen, in denen die Poilus weder die deutsch-feindlichen chansons revanchardes noch die fern der Front geschriebenen Soldatenlieder singen, sondern bekannte Heimat- und 
Liebeslieder anstimmen. Erst nach seiner Entlassung aus dem Militärdienst singt ein Kriegsinvalide in einem Pariser Café das zum Repertoire der chansons revanchardes gehörende Lied Le rêve passe ${ }^{4}$.

Trotz ihrer geringen Popularität bei den Poilus seien hier zunächst einige der in den ersten Jahren der Grande Guerre entstandenen heroisierenden Chansons Pariser Autoren vorgestellt:

In seinem Chanson Le cri du poilu fragt Vincent Scotto 1915 sein Pariser Publikum, was den Poilus wohl am meisten fehle, wenn sie nicht gerade kämpfen müssten. Die Antwort lautet: Une femme, une femme! Der Gedanke an eine Frau, die sie vermissen, verließe die Soldaten nur während des Gefechts. Scotto verharmlost das Elend des Krieges. Sein vorwiegend weibliches Publikum hört nur, dass die Soldaten lieber bei ihren Frauen wären als an der Front auf die Deutschen schießen zu müssen.

Das Chanson Ce que chantent les flots de la Marne erinnert an die siegreiche Marneschlacht, die im Herbst 1914 den deutschen Blitzkrieg beendet. Pierre Willems besingt in seinem 1915 publizierten Lied zunächst die Marne rieuse, dann die Marne rougie. Das in Friedenszeiten lachende Wasser des Flusses rötet sich während der Schlacht. Willems behauptet, dass sich Deutschland seit dem Ende des Deutsch-Französischen Krieges auf den nächsten Konflikt mit Frankreich militärisch vorbereitet habe. Das seit 1871 in Frankreich vorhandene Streben nach einer Revanche verschweigt er. Willems beantwortet vorzeitig die Frage der Kriegsschuld, die der Versailler Vertrag nur Deutschland zuschreiben wird.

Im Kriegsjahr 1916 widmen die Autoren Jack Cazol und Eugène Joullot ihr Chanson Verdun! On ne passe pas ! dem für Franzosen wie Deutsche blutigsten Kriegsschauplatz des Ersten Weltkrieges. In dem Chanson dieser Autoren stehen sich nicht nur die Soldaten beider Länder als erbitterte Feinde gegenüber, sondern auch die allegorischen Figuren, die Deutschland und Frankreich in vielen Karikaturen repräsentieren: der schwarze preußische Adler und der gold-farbene gallische Hahn. Das an Feindbildern reiche Lied endet mit den boshaften Zeilen: « Pendant que nous chantons La Marseillaise, / Les assassins fuient devant les soldats. » Die ihre Nationalhymne singenden Franzosen sind Soldaten, die vor ihnen fliehenden Feinde Mörder! Mit dieser Diffamierung wird den Deutschen der Soldatenstatus aberkannt.

Die Allegorie des gallischen Hahnes, der den preußischen Adler bekämpft, verwendet auch Théodore Botrel in seinem 1916 geschriebenen Chanson Les Coqs d'Or. Botrel wendet sich an die goldenen Hähne, die als Wetterhähne auf den Spitzen französischer Kirchtürme zu sehen sind. Er fordert die Hähne auf, ihre Kirchtürme zu verlassen, denn die Stunde nahe, in der sich der erschöpfte räuberische Adler fast tot ihren Sporen ausliefern werde.

Das Chanson La Mimi, das Théodore Botrel 1916 verfasst, ist weit weniger „poetisch“ als das vorige Lied: Der Schütze eines Maschinengewehrs macht seiner Waffe, einer mitrailleuse, die er ma p'tite Mimi nennt, eine

\footnotetext{
${ }^{4}$ Roland Dorgeles, Les Croix de bois, Paris, Albin Michel, 1919, S. 275.
} 
Liebeserklärung. Auch wenn ihn seine Rosalie daheim liebevoll anschaue, sei es doch Mimi, die er am meisten liebe. Er liebe es, wenn seine $p$ 'tite Mimi auf ihre Weise sänge. Wenn die Boches sich ihnen näherten, begänne das Konzert. Dann würde er die Deutschen mit dem Feuer seines Maschinengewehrs niedermähen.

In seinem im November 1916 erschienenen Antikriegsroman Le feu, der auf eigenen Fronterlebnissen beruht, kritisiert Henri Barbusse den Missbrauch der Chansons: "Ceux qui s'enivrent avec la musique militaire ou avec les chansons versées au peuple comme des petits verres... ${ }^{5}$ "Insbesondere denunziert Barbusse die Aufopferung der Völker: « les peuples entiers vont à la boucherie, rangés en troupeaux d'armées, pour qu'une caste galonnée d'or écrive ses noms de princes dans l'histoire; pour que des gens dorés aussi, qui font partie de la même gradaille, brassent plus d'affaires... ${ }^{6}$ » Hier wird die Diskrepanz zwischen den heroisierenden Liedern der Pariser Autoren und dem täglichen Überlebenskampf und Empfinden der Poilus deutlich.

Die ersten Anzeichen eines Nachlassens des Heldenmutes, des Gehorsams und der Opferbereitschaft der Poilus beschreiben in ihren Romanen sowohl Henri Barbusse als auch Roland Dorgelès. Beide Autoren erleben die Exekution eines wegen Feigheit vor dem Feinde von einem Militärgericht zum Tode verurteilten Soldaten. Bei Dorgelès findet die Exekution des Verurteilten vor seinem in voller Mannschaftsstärke angetretenen Regiment statt $^{7}$. Als das Regiment im Marschschritt die Hinrichtungsstätte verlässt, spielt die Regimentsmusik das Lied Mourir pour la Patrie ${ }^{8}$. Der Refrain dieses Liedes glorifiziert die Opferbereitschaft der Soldaten: « Mourir pour la Patrie / Mourir pour la Patrie / C'est le sort le plus beau, le plus digne d'envie / C'est le sort le plus beau, le plus digne d'envie. » Dorgelès prangert in dem dieser Hinrichtung gewidmeten Kapitel seines Romans den Zynismus der Militärführung gegenüber den Kameraden des exekutierten Soldaten an.

Im Kriegsjahr 1917 sinkt die Truppenmoral. Trotz der enormen Verluste ändert sich die Kriegslage nicht. Es kommt zu Meutereien. Immer mehr Soldaten fragen sich, welchen Sinn ihr Opfer haben soll. Der Hass der Poilus richtet sich bald mehr gegen die Kapitalisten, die fern der Front ihren Wohlstand genießen, als gegen die Deutschen. In dieser kritischen Situation beginnen die Soldaten selbst gedichtete Chansons zu singen. Im Unterschied zu den Kriegsliedern der Pariser Autoren, die von den Zivilisten mehr geschätzt werden als von den Poilus, sind die Texte dieser Chansons

${ }^{5}$ Henri BARBUSSE, Le Feu, Paris, Flammarion, 1965, S. 371.

${ }^{6}$ Ibid., S. 369.

${ }^{7}$ Roland Dorgeles, Les Croix de bois, Paris, Albin Michel, 1919, S. 149 f.

${ }^{8}$ Der eigentliche Titel dieses Liedes lautet Chœur des Girondins. Der Text wurde 1847 von Alexandre Dumas und Auguste Maquet anlässlich einer Theateraufführung ihres gemeinsam verfassten Romans Le Chevalier de Maison-Rouge geschrieben und von Alphonse Varney vertont. Der dazugehörige Refrain Mourir pour la Patrie stammt dagegen aus dem 1792 von Claude-Joseph Rouget de Lisle kurz nach der Marseillaise gedichteten und vertonten Kriegslied Roland à Roncevaux. Als Chant des Girondins war das Lied von 1848 bis 1851 die Nationalhymne der Zweiten Republik. 
defätistisch. Die französische Armeeführung untersagt das Singen solcher Lieder. Sie verspricht, jeden Hinweis, der zur Festnahme ihrer Autoren führt, zu belohnen. Obwohl die Generalität hohe Geldsummen und die sofortige Entlassung aus der Armee anbietet, denunziert keiner der Poilus die dichtenden Kameraden. Die Autoren der defätistischen Chansons bleiben anonym.

Das wohl bekannteste dieser Lieder ist La chanson de Craonne, das Paul Vaillant-Couturier, der spätere Chefredakteur der kommunistischen Tageszeitung L'Humanité, als Unteroffizier an der Front hört, aufschreibt und nach dem Krieg veröffentlicht. Das Chanson beschreibt die Verzweiflung der Soldaten, die ein von den Deutschen gehaltenes Hochplateau erstürmen sollen, das in der Nähe des Ortes Craonne im nordfranzösischen Département Aisne liegt: «Adieu la vie, adieu l'amour, / Adieu toutes les femmes. / C'est bien fini, c'est pour toujours, / De cette guerre infâme. / C'est à Craonne, sur le plateau, / Qu'on doit laisser sa peau, / Car nous sommes tous condamnés, / C'est nous les sacrifiés!». Die Soldaten sagen dem Leben, der Liebe und den Frauen Adieu. Da sie den Tod erwarten, verabschieden sie sich zugleich von diesem abscheulichen Krieg. Auf dem Plateau von Craonne werden sie fallen, denn sie sind alle zum Tode verurteilt. Dann aber wendet sich das Chanson anklagend denjenigen zu, für die sie sterben sollen: den Reichen, die in Paris das Leben genießen. Die Soldaten fordern diese Herren auf, in die Schützengräben zu kommen und ihren Reichtum selbst zu verteidigen, denn sie besäßen nichts und wollten nicht länger kämpfen: « Ce sera votre tour, messieurs les gros, / De monter sur le plateau, / Car si vous voulez la guerre, / Payez-la de votre peau !».

Das Lied, dessen Autor trotz einer Belohnung von einer Million Franken nicht gefasst wird, ruft zu Meuterei und Klassenkampf auf: Wenn die Reichen den Krieg wollten, dann sollten sie ihn auch mit ihrem eigenen Leben bezahlen! Wegen dieser gesellschaftskritischen Botschaft wird das Chanson nach dem Ersten Weltkrieg in das traditionelle Liedrepertoire der politischen Linken aufgenommen.

In der Reihe der bisher vorgestellten Lieder ist La chanson de Craonne das einzige Lied, das keine antideutschen Ressentiments weckt. Außerdem ist es eines der wenigen Chansons des Zeitraumes 1871 bis 1919, die auch heute noch in Frankreich bekannt sind: Der Regisseur Fabrice Cazeneuve integriert es in seinen 2002 gedrehten Fernsehfilm La Dette; der bekannte Sänger Maxime Le Forestier nimmt das Lied 2003 in sein Repertoire auf; ein zum Tode Verurteilter singt La chanson de Craonne in dem Spielfilm Un long dimanche de fiançailles, den der Regisseur Jean-Pierre Jeunet 2004 produziert.

Während der Meutereien entsteht ebenfalls das Chanson Non, non, plus de Combats eines anonymen Autors. Das pazifistische Lied verurteilt den Krieg, der ein einziges Gemetzel sei: La guerre est une boucherie. Der Autor fragt, ob der Arbeiter seine Fabrik verlassen müsse, um im Krieg vor die Wahl gestellt zu werden, entweder sein Leben zu verlieren oder seinen 
Nächsten zu töten. Die Völker sollten einander lieben, die Brüder einander nicht mehr töten: „Aimons-nous, peuples d'ici-bas, / Ne nous tuons plus entre frères !". Ohne sie beim Namen zu nennen, werden die Deutschen zu Brüdern erklärt! Sie sind nicht länger feindliche Soldaten, sondern Arbeiter, die an der Front das gleiche Schicksal ertragen müssen wie die Franzosen. Das pazifistische Chanson wird verboten, weil es ein Ende des Krieges fordert und dem Feindbild der staatlichen Propaganda widerspricht.

Im Kriegsjahr 1917 beteiligen sich etwa 40.000 Poilus an den Meutereien. General Philippe Pétain, der „Sieger von Verdun“, stellt innerhalb weniger Wochen die Ordnung wieder her: Um ein Exempel zu statuieren, wird jeder zehnte Meuterer vor ein Kriegsgericht gestellt. Ein Losverfahren ermittelt die Angeklagten. Von 3.427 Urteilen entfallen 554 auf die Todesstrafe. Letztendlich werden 49 Meuterer exekutiert ${ }^{9}$. Gleichzeitig sorgt Pétain aber auch dafür, dass die Soldaten bessere Nahrung und häufiger Fronturlaub erhalten. Diese Maßnahmen tragen zur Popularität des Generals bei, der 1918 zum Marschall befördert wird.

Ähnlich wie die anonymen Autoren der defätistischen Chansons äußert sich - allerdings fern der Front - im Oktober 1916 auch der deutsche Kriegsdienstverweigerer und Anarchist Erich Mühsam in seinem Soldatenlied 1916, in dem es unter anderem heißt: „Soldaten! Ruft's von Front zu Front: Es ruhe das Gewehr! Wer für die Reichen bluten konnt, kann für die Seinen mehr. Ihr drüben! Auf zur gleichen Pflicht! Vergesst den Freund im Feinde nicht!“.

\section{Waffenstillstand 1918 und Versailler Vertrag 1919}

Im Herbst 1918 bricht die deutsche Westfront zusammen. Der Waffenstillstand wird am 11. November 1918 unterzeichnet. Der Versailler Vertrag besiegelt 1919 die Rückkehr Elsass-Lothringens und seiner Bevölkerung nach Frankreich. Fast fünfzig Jahre nach der Veröffentlichung des Chansons Vous n'aurez pas l'Alsace et la Lorraine! erfüllt sich die Revanche für die 1870/71 erlittene militärische Niederlage. Das 1919 von Charles Louis Pothier verfasste Chanson Ils ont rendu l'Alsace et la Lorraine gibt den Jubel wieder, der im Elsass nach vier Kriegsjahren in weiten Teilen der Bevölkerung tatsächlich herrscht ${ }^{10}$. Das harte Militärregime der Deutschen ist beendet.

Der Refrain des Chansons von 1919 knüpft an das im Refrain seines Pendants aus dem Jahre 1871 gegebene Versprechen an, dass die Elsässer immer Franzosen bleiben würden. Fast fünfzig Jahre später stellt Pothier im Refrain seines Chansons triumphierend fest, dass das Herz des Elsass

\footnotetext{
9 Pierre SAKA, Y'a d'la France en chansons, Paris, Larousse, 2001, S. 49.

10 Alfred WAHL, Jean-Claude RICHEZ, L'Alsace entre France et Allemagne, Paris, Hachette, 1994, S. 252.
} 
französisch geblieben sei. Die Deutschen, die Frankreich verspottend immer „Niemals“ sagten, hätten endlich das Elsass und Lothringen zurückgegeben. Nicht Liebe, nur Hass hätten sie dort entfachen können: «Ils ont enfin rendu l'Alsace et la Lorraine, / Eux qui raillaient la France et qui disaient "jamais !" / Ils n'avaient su là-bas que déchaîner la haine, / Mais le cœur de l'Alsace était resté français, / Oui, le cœur de l'Alsace était resté français. »

\section{Retrospektive Chansons}

Abgesehen von einigen Liedern zu Ehren der französischen Generale Foch, Joffre und Pétain, in denen der besiegte deutsche Feind ebenso wenig erwähnt wird wie in den Liedern, deren Helden die legendären Poilus sind, entstehen nach dem Kriegsende keine Chansons, die den Ersten Weltkrieg thematisieren. Das Chanson der Nachkriegszeit überlässt der Literatur die Verarbeitung der Tragödie des Ersten Weltkrieges: So erscheint im Jahr 1929 der Roman Im Westen nichts Neues von Erich Maria Remarque, der 1917 Soldat an der Westfront war. Sein Roman wurde 1930 in den USA von Lewis Milestone verfilmt, die Vorführung des Films in Deutschland aber von den Nationalsozialisten gestört und ab 1933 verboten. Humphrey Cobb, der im Ersten Weltkrieg drei Jahre lang in einem kanadischen Regiment diente, schrieb 1935 den Roman Paths of Glory, der 1958 in den USA von Stanley Kubrick verfilmt wurde. Da dieser Film in Frankreich als Angriff auf die Ehre der französischen Armee empfunden wurde, durfte er dort erst ab 1975 gezeigt werden.

Im Chanson erfolgt der Rückblick auf die Grande Guerre erst nach dem Zweiten Weltkrieg. Der zeitliche Abstand zum Ersten Weltkrieg ist so groß, dass keiner der drei Autoren dieser retrospektiven Chansons die Katastrophe der Jahre 1914 bis 1918 selbst erlebt hat: Georges Brassens wurde 1921, Jacques Brel 1929 und Michel Sardou 1947 geboren. Die Auseinandersetzung dieser drei auteurs-compositeurs-interprètes mit dem Ersten Weltkrieg könnte kaum gegensätzlicher sein: Brassens verspottet 1956 mit La guerre de 14-18 den Totenkult der Kriegsveteranen; Brel erinnert 1977 mit Jaurès an den 1914 ermordeten Pazifisten Jean Jaurès; Sardou gedenkt 1979 mit Verdun der Gefallenen der Grande Guerre. Keines dieser Chansons ist dem Heldentum der Poilus oder den Meutereien des Jahres 1917 gewidmet. Auch knüpft keiner der drei Autoren mit seinen Chansons an die antideutschen Ressentiments an, die bei Ausbruch des Ersten Weltkrieges vorherrschten.

\section{Fazit}

In Deutschland gehörte ein frankophobes, in Frankreich ein germanophobes Liedgut über viele Generationen hinweg zum Selbstverständnis beider Nationen. Feindbilder erleichterten die Definition 
der jeweiligen nationalen Identität. Auf französischer Seite stieß die identitäts-stiftende und die Konfliktbereitschaft unterhaltende und verschärfende Wirkung deutsch-feindlicher Lieder spätestens mit den Meutereien des Jahres 1917 an ihre Grenzen. Diese Feststellung trifft ebenso auf das Repertoire der chansons revanchardes zu wie auf die von Pariser Autoren fern der Front verfassten heroisierenden Chansons der ersten Jahre der Grande Guerre. Solche Lieder wurden zwar in den Pariser cafés-concerts, jedoch nicht an der Front gesungen. Das Publikum dieser Etablissements bestand kaum aus Frontsoldaten auf Heimaturlaub, sondern zunehmend aus Personen, die vom Krieg finanziell profitierten, Militärs, die nicht unmittelbar an der Front kämpfen mussten, und einer Bourgeoisie, die keine Vorstellung vom grauenhaften Frontalltag der Poilus hatte bzw. haben wollte. Es waren diejenigen, die in den defätistischen Chansons der Meuterer von 1917 als eigentliche Feinde entlarvt wurden : Die Kapitalisten, die Offizierskaste und das wohlhabende Bürgertum. Dieses neue, über die deutsch-französische Grenze hinweg reichende Feindbild thematisieren auch Henri Barbusse und Roland Dorgelès in ihren Antikriegsromanen.

Chronologie der zitierten Lieder

\section{Erste Feindbilder}

- Der Gott, der Eisen wachsen ließ (Ernst Moritz Arndt, 1812)

- Rheinlied (Nikolaus Becker/Robert Schumann, 1840)

- Die Wacht am Rhein (Max Schneckenburger, 1840)

- Lied der Deutschen (Hoffmann von Fallersleben/Joseph Haydn, 1841)

- Heil dir im Siegerkranz (Balthasar Gerhard Schumacher, 1793/1871)

- La Marseillaise (Claude Joseph Rouget de Lisle, 1792)

- Le Chant du Départ (Étienne-Nicolas Méhul und Marie-Joseph Chénier, 1794)

\section{Chansons revanchardes}

- Vous n'aurez pas l'Alsace et la Lorraine! (Gaston Villemer, 1871)

- Les Cuirassiers de Reichshoffen (Gaston Villemer, 1871)

- Le Clairon (Paul Déroulède, 1875)

- La fiancée alsacienne (Gaston Villemer, 1881)

- Le fils de l'Allemand (Gaston Villemer und Lucien Delormel, 1882)

- Le violon brisé (René de Saint-Prest, 1885)

- En revenant de la revue (Lucien Delormel, 1886) 
- Le Père la Victoire (Lucien Delormel, 1889)

- Le Rêve passe (Armand Foucher, 1906)

\section{Chansons der Grande Guerre}

- Le cri du poilu (Vincent Scotto, 1915)

- La Mimi (Théodore Botrel, 1916)

- Ce que chantent les flots de la Marne (Pierre Willems, 1915)

- Verdun! On ne passe pas ! (Jack Cazol und Eugène Joullot, 1916)

- Les Coqs d'Or (Théodore Botrel, 1916)

- La chanson de Craonne (Anonym, 1917)

- $\quad$ Non, non, plus de Combats (Anonym, 1917)

- $\quad$ Soldatenlied 1916 (Erich Mühsam, 1916)

\section{Waffenstillstand 1918 und Versailler Vertrag 1919}

- Ils ont rendu l'Alsace et la Lorraine (Charles Louis Pothier, 1919)

\section{Retrospektive Chansons}

- $\quad$ La guerre de 14-18 (Georges Brassens, 1956)

- Jaurès (Jacques Brel, 1977)

- Verdun (Michel Sardou, 1979) 